
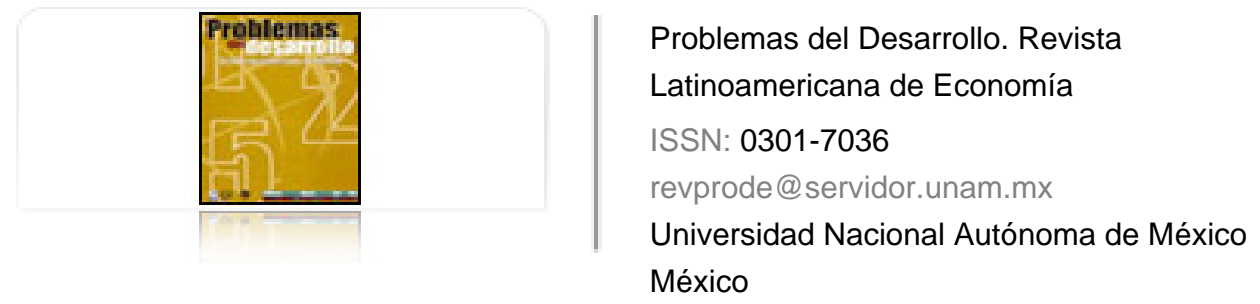

Medialdea, Bibiana

Límites estructurales al desarrollo económico: Brasil (1950-2005)

Problemas del Desarrollo. Revista Latinoamericana de Economía, vol. 43, núm. 171, octubre-

diciembre, 2012, pp. 55-81

Universidad Nacional Autónoma de México

Distrito Federal, México

Disponible en: http://www.redalyc.org/articulo.oa?id=11823951004

- Cómo citar el artículo

- Número completo

- Más información del artículo

Página de la revista en redalyc.org

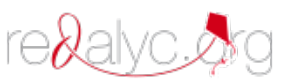

Sistema de Información Científica

Red de Revistas Científicas de América Latina, el Caribe, España y Portugal Proyecto académico sin fines de lucro, desarrollado bajo la iniciativa de acceso abierto 


\title{
LÍMITES ESTRUCTURALES AL DESARROLLO ECONÓMICO: BRASIL (I950-2005)
}

\begin{abstract}
Bibiana Medialdea*
Fecha de recepción: 27 de noviembre de 2011. Fecha de aceptación: 13 de abril de 2012.
\end{abstract}

\begin{abstract}
RESUMEN
Este artículo plantea que la reproducción del subdesarrollo brasileńo se explica por la persistencia de un conjunto de elementos que operan como obstáculos de carácter estructural: los denominamos límites estructurales al desarrollo económico. En primer lugar, se plantea una propuesta interpretativa que articula las principales variables que intervienen en la dinámica económica favoreciendo o dificultando su proceso de acumulación. Posteriormente, se analizan las condiciones específicas de la economía brasileña durante el periodo 1950-2005. Se atiende tanto a las debilidades productivas de la economía brasileña, como a su demanda agregada y la distribución de la renta. En conclusión, estos factores determinan una restricción respecto a su ahorro interno. La interrelación de dichos límites explica la reproducción del subdesarrollo en el país.
\end{abstract}

Palabras clave: límites estructurales al desarrollo económico, proceso de acumulación, Brasil.

\section{Structural Limits on Economic Development: BraziL (I950-2005)}

\begin{abstract}
This article proposes that the reproduction of Brazilian underdevelopment is explained by a set of elements that operate as structural obstacles: we call them structural limits on economic development. First, an interpretative proposal is set forth to articulate the key variables that affect economic dynamics that either favor or hinder accumulation processes. Later, the specific circumstances of the Brazilian economy in the period from 1950-2005 are analyzed. The article touches on both the Brazilian economy's productive weaknesses as well as aggregate demand and income distribution. To conclude, these factors determine a restriction on internal savings. The relationship between these limits explains the reproduction of underdevelopment in the country.
\end{abstract}

Key Words: structural limits on economic development, accumulation process, Brazil.

* Profesora de la Universidad Complutense de Madrid, España. Correo electrónico: bibiana@ccee.ucm.es 


\section{LIMITES STRUCTURELLES AU DÉVELOPPEMENT ÉCONOMIQUE: LE BRÉSIL (1950-2005) \\ Résumé}

Cet article pose que la reproduction du sous-développement brésilien s'explique par la persistance d'un ensemble d'éléments qui agissent comme des obstacles de caractère structurel: les dénommés limites structurelles au développement économique. En premier lieu, il est fait une proposition interprétative qui articule les principales variables qui interviennent dans la dynamique économique favorisant ou entravant son processus d'accumulation. Ensuite, sont analysées les conditions spécifiques de l'économie brésilienne durant la période 1950-2005. Il est pris en considération tant les faiblesses productives de l'économie brésilienne que sa demande ajoutée et la distribution de la rente. En conclusion, ces facteurs déterminent une restriction par rapport à l'épargne interne. L'interconnexion desdites limites explique la reproduction du sousdéveloppement dans le pays.

Mots clés: limites structurelles au développement économique, processus d'accumulation, Brésil.

\section{OS LIMITES ESTRUTURAIS AO DESENVOLVIMENTO ECONÔMICO: BRASIL (1950-2005) \\ Resumo}

Este artigo planteia que a reprodução do subdesenvolvimento brasileiro se explica pela persistência de um conjunto de elementos que operam como obstáculos de caráter estrutural: os chamamos de limites estruturais do desenvolvimento econômico. Em primeiro lugar, se planteia uma proposta interpretativa que articula as principais variáveis que intervêm na dinâmica económica favorecendo ou dificultando seu processo de acumulação. Posteriormente, se analisam as condiçôes especificas da economia brasileira durante o período 1950-2005. Atende-se tanto as debilidades produtivas da economia brasileira, como a sua demanda agregada e a distribuição da renda. Em conclusão, estes fatores determinam uma restrição no que diz respeito a sua poupança interna. A interrelaçâo de ditos limites explica a reprodução do subdesenvolvimento no país.

Palavras-chave: limites estruturais ao desenvolvimento econômico, processo de acumulação, Brasil.

\section{经济发展的结构约束：巴西的案例（1950-2005） \\ 摘要 \\ 本文认为, 巴西欠发达的再生可通过一系列的结构性障碍因素来解释, 可 称之为经济发展的结构约束。首先, 提出确立影响经济动态的关键性解释 变量, 促进或阻碍积累过程。其次, 分析了1950-2005年巴西经济的具体 环境。本文涉及了巴西经济生产脆弱性以及总需求和收入分配等方面。结 论认为, 这些因素对内部储蓄具有制约作用。这些制约因素之间的关系可 以解释巴西欠发达的可再生性。 \\ 关键词: 经济发展的结构制约 积累过程巴西}




\section{INTRODUCCIÓN}

A lo largo del siglo xx Brasil ha registrado transformaciones económicas y sociales de gran importancia. Sin embargo, las condiciones de subdesarrollo que caracterizan a su economía se han seguido reproduciendo. ${ }^{1}$ Este artículo plantea que la reproducción del subdesarrollo brasileño se explica por la persistencia de un conjunto de elementos que operan como obstáculos de carácter estructural, y en cuya presencia es inviable superar la situación de subdesarrollo. Denominamos a este conjunto de elementos límites estructurales al desarrollo económico. En el apartado inicial se establecen los fundamentos básicos de la propuesta interpretativa, es decir, la articulación de las principales variables que intervienen en la dinámica económica favoreciendo o dificultando su proceso de acumulación. Los apartados siguientes se centran en las condiciones específicas de la economía brasileña durante el periodo $1950-2005 .{ }^{2}$ Atendemos primero a las debilidades productivas que determinan un limitado proceso de acumulación y una productividad del trabajo reducida. Posteriormente, consideramos los elementos relacionados con la demanda agregada y la distribución de la renta que explican dicha limitación. En conclusión, dichos factores determinan una insuficiencia de ahorro interno, así como la propensión a darle un uso improductivo. La interrelación de dichos límites explica, por tanto, la reproducción del subdesarrollo en el país.

\section{APROXIMACIÓN ANALÍTICA A LA REPRODUCCIÓN DEL SUBDESARROLLO. LOS LÍMITES ESTRUCTURALES AL DESARROLLO ECONÓMICO}

La cuestión del (sub)desarrollo es una de las temáticas más amplias y controvertidas de las que se ocupa el análisis económico. ${ }^{3}$ Este trabajo no pretende en absoluto tratarla en su amplitud. Se limita a formular una propuesta interpretativa

1 Lo cual no es inconveniente para que Brasil presente algunos indicadores económicos con niveles superiores al promedio de las economías subdesarrolladas.

2 Como consecuencia, el análisis realizado no es automáticamente extrapolable a cualquier economía subdesarrollada.

3 Una panorámica de las aportaciones y controversias fundamentales se puede encontrar en Todaro (1985), Griffin (1989), Szirmay (2005) y Puerto (2008). 
que permita explicar cómo, en una economía no desarrollada, se producen sucesivos vínculos estructurales que reproducen su subdesarrollo.

Para ello, se establece una delimitación conceptual que compara cómo interactúan determinadas variables económicas fundamentales en una economía desarrollada y una subdesarrollada. Comúnmente, la forma más inmediata o intuitiva que se utiliza para realizar dicha distinción es la utilización de ciertos indicadores globales, como el PIB per cápita. Aunque dicho indicador presenta notables debilidades, veremos que también proporciona información significativa sobre la economía brasileña y su evolución.

En términos analíticos, la primera aproximación que cabe hacer para establecer la distinción entre desarrollo y subdesarrollo es a partir de la economía política clásica, y en particular de las propuestas de Smith, Ricardo, y fundamentalmente Marx, sobre el proceso de acumulación. En primera instancia, la capacidad de acumulación viene definida por la magnitud del stock de capital y por su estructura interna. Por un lado, la dotación de capital es el determinante fundamental de las posibilidades de crecimiento sostenido de la producción. Por tanto la dimensión del stock es un factor decisivo para el desarrollo, y se convierte en un obstáculo estructural para las economías que carecen de un nivel suficiente, y que por ello se ven obligadas a realizar un gran esfuerzo inversor para conseguir ampliar su capacidad de acumulación. Por otra parte, la composición técnica del stock de capital determina la estructura sectorial de la actividad productiva. Históricamente, el proceso de transformación que han registrado las economías desarrolladas se ha basado en la industrialización y, en particular, en el peso creciente de los bienes de capital como componente cada vez más importante dentro de la producción. Así, tanto el peso relativo de la industria dentro de la economía, como el de las ramas de bienes de capital dentro de la estructura industrial, son elementos fundamentales que, en interacción, determinan la capacidad de una economía para reproducir de forma ampliada su dinámica de acumulación.

Por tanto, dicha dinámica presenta una doble vertiente. Es un proceso cuantitativo, ya que la acumulación implica una mayor dimensión del stock, sobre todo de capital no residencial y, dentro de él, de máquinas y otros equipos productivos. Y a la vez es un proceso cualitativo, sustentado en que ese capital incorpora sistemáticamente un mayor progreso técnico. De ello se deriva que la dinámica de acumulación de una economía desarrollada genera un persistente aumento de la productividad, ya que implica un paulatino incremento del coeficiente capital-trabajo $(\mathrm{K} / \mathrm{L})$ a la vez que una reducción del coeficiente capital-producto $(\mathrm{K} / \mathrm{Y})$, es decir, una mejora de los dos componentes en los que 
se descompone el aumento de la productividad del trabajo. ${ }^{4}$ Éste es otro indicador crucial que distingue a las economías no desarrolladas: su reducido nivel de productividad y la lentitud con la que se incrementa a lo largo del tiempo.

Así, desde el punto de vista de la dinámica de acumulación cabe destacar un conjunto de variables relacionadas con la capacidad de producción, es decir, con la oferta, que distinguen con claridad el dispar comportamiento de una economía no desarrollada con respecto a otra desarrollada: reducido nivel de PIв per cápita, escasa magnitud del stock de capital, mínima presencia de bienes de capital, y baja productividad del trabajo.

Ahora bien, la propia experiencia de las economías desarrolladas pone de manifiesto que esa dinámica de acumulación no es continuada, sino que sigue una evolución cíclica a lo largo del tiempo. La capacidad de producir (producción potencial) se hace real (producción efectiva) cuando existe un mercado y unas perspectivas de rentabilidad que estimulan las expectativas empresariales. Surge, pues, la necesidad de incorporar nuevos elementos analíticos para explicar la dinámica de crecimiento económico, elementos que conciernen principalmente a la demanda agregada y a la distribución de la renta, y que a su vez se relacionan con el funcionamiento de las instituciones y el contexto sociopolítico. A este respecto, se constatan nuevas y decisivas diferencias entre lo que acontece en una economía subdesarrollada por contraste con otra desarrollada.

Podemos fundamentar teóricamente esas cuestiones partiendo de la importancia crucial de la inversión en el funcionamiento económico, y atendiendo al doble impacto que ejerce sobre la dinámica económica, como componente de la demanda y como determinante de la capacidad productiva (Kalecki, 1977; Palazuelos y Fernández, 2009). De un lado, como componente fundamental de la demanda efectiva, la inversión influye de forma determinante sobre la evolución de variables como los beneficios, la producción y el empleo. Por otra parte, la inversión ejerce un efecto directo sobre la capacidad productiva potencial, ya que hace posible que se incremente el stock de capital. Simultáneamente, los factores que determinan el comportamiento de la inversión son ciertamente plurales, puesto que tienen que ver con las expectativas de rentabilidad, que dependen de elementos dispares.

De ese modo, la dinámica económica se configura mediante la interacción de un cúmulo de variables que en las fases ascendentes del ciclo generan sinergias favorables al crecimiento, entre la demanda y la oferta

4 La productividad del trabajo $(\mathrm{Y} / \mathrm{L})$ indica la relación entre $(\mathrm{K} / \mathrm{L})$ y $(\mathrm{K} / \mathrm{Y})$, por lo que si a, b y $\mathrm{c}$ son las tasas respectivas de crecimiento, tenemos que $\mathrm{a}=\mathrm{b}-\mathrm{c}$. 
(inversión-producción-demanda agregada-inversión) y entre demanda y distribución de la renta (inversión-beneficios-expectativas-inversión). Además, esos círculos virtuosos se generan en contextos sociales y políticos determinados que afectan positiva o negativamente a sus mecanismos reproductivos. La conformación misma de la tasa de rentabilidad es resultado del conflicto que en toda sociedad capitalista se genera en torno a la división de la renta entre beneficios y salarios. Así, los intereses políticos existentes y su capacidad para imponerse, son de crucial importancia.

También desde esta perspectiva que vincula el proceso de acumulación con la demanda agregada, la distribución de la renta y las condiciones sociopolíticas, se aprecian diferencias notorias en las economías subdesarrolladas. En términos históricos, la debilidad secular de la inversión es lo que determina la escasez de stock de capital y su escaso contenido tecnológico. En términos de dinámica económica, el comportamiento de la inversión y del conjunto de la demanda agregada de las economías subdesarrolladas se explica fundamentalmente por las condiciones de la distribución de la renta, firmemente reforzadas por el marco político vigente. La tasa de inversión es modesta porque los grupos sociales que detentan la mayor parte de la renta la utilizan con otros propósitos. El consumo privado también es escaso porque la mayoría de la población carece de capacidad adquisitiva. A su vez, la reducida inversión productiva y la estrechez del mercado de consumo hacen que los niveles de beneficio empresarial no sean atractivos para realizar mayores inversiones y, por tanto, para fortalecer la capacidad productiva de la economía. De ese modo, el proceso de acumulación queda obstaculizado porque se reproduce sistemáticamente un nudo de relaciones entre oferta, demanda y distribución de la renta, los denominados límites estructurales al desarrollo económico, que consolidan su subdesarrollo y que resultan antagónicos con la mecánica de acumulación que registran las economías desarrolladas.

\section{DEBILIDAD PRODUCTIVA Y LIMITADA CAPACIDAD DE ACUMULACIÓN}

Según adelantamos en el apartado anterior y como punto de partida, el indicador del Рів per cápita brinda una primera información relevante sobre la economía brasileña. A la altura de 1950 la renta media de su población equivalía aproximadamente a un tercio de la renta per cápita de los países europeos más avanzados como Francia y Holanda, situándose mucho más próxima, entre el 
$75 \%$ y el $87 \%$, de la de Portugal, Grecia o España. ${ }^{5}$ En el transcurso de la segunda mitad del siglo xx se han profundizado dichas diferencias, de forma que al final del siglo la renta per cápita brasileńa acumula una distancia abismal con todos los países europeos. No sólo equivale apenas al 25-30\% de la renta per cápita de los países más potentes de la región como Francia, Alemania, Holanda e Italia, sino que también se sitúa en sólo el 35-45\% de la renta media de los países europeos respecto a los que Brasil se encontraba relativamente "cercano" a mediados de siglo. La evolución es inequívoca y la comparación con España resulta ilustrativa: en esa segunda mitad de siglo la proporción del рів per cápita brasileño se ha reducido desde el $75 \%$ hasta el 36\% respecto al español.

Este diferencial se ha ensanchado a pesar de que entre 1950 y 1980 Brasil registra un crecimiento económico potente y duradero, que supone un importante impulso industrializador, y con él, cambios estructurales de cierta envergadura. Dicha dinámica se interrumpe drásticamente a partir de los ańos ochenta. En cualquier caso, a lo largo de las décadas la estructura productiva brasileña arrastra debilidades que limitan su dinámica de acumulación. En particular, registra una precariedad manifiesta en las ramas que producen bienes de capital, un stock de capital muy escaso y un crecimiento claramente insuficiente de la productividad del trabajo, es decir, tres características que definimos en el apartado anterior como parte de los límites estructurales al desarrollo.

El esquema de funcionamiento económico que durante tres décadas permite a Brasil no sólo obtener tasas de crecimiento elevadas, sino también impulsar un significativo proceso de industrialización se corresponde, a grandes rasgos, con la estrategia denominada Industrialización por Sustitución de Importaciones (ISI) que en la época teorizan la Comisión Económica para América Latina y El Caribe (CEPAL) y el brasileño Banco Nacional do Desenvolvimento (BNDES). ${ }^{6}$ Dicha estrategia se basa en un acelerado y amplio proceso de industrialización que se dirige y dinamiza desde el Estado y se concentra en el mercado interno. La industria se convierte así en el motor del crecimiento y en el centro de transformaciones económicas y sociales de hondo calado. En palabras de Carlos Lessa "[...] la industrialización, entendida como la construcción de un sistema industrial, es la columna vertebral del proceso nacional-desarrollista inaugurado en 1930 y que durante medio siglo sería el proyecto nacional brasileño" (Lessa, 2005: 18).

Los datos que se facilitan en este párrafo se han calculado a partir de Maddison (2002).

6 Sobre la estrategia IsI se puede consultar Tavares (1964), Rodríguez (1993) y CEPAL (1998). 
Como resultado, la estructura sectorial se transforma sensiblemente. Las actividades agrícolas y las industriales, como recoge la Gráfica 1, pasan de tener aproximadamente el mismo peso relativo sobre la producción en 1950, en torno a un $24 \%$, a perder y ganar, respectivamente, del orden de 15 puntos porcentuales. En 1980 el sector primario es sólo un 10.1\% de la producción total, mientras la industria concentra un $40.9 \%$. Los servicios, por su parte, mantienen grosso modo su participación.?

Gráfica 1. Estructura sectorial de la producción: 1950-2005

(\% del PIB)

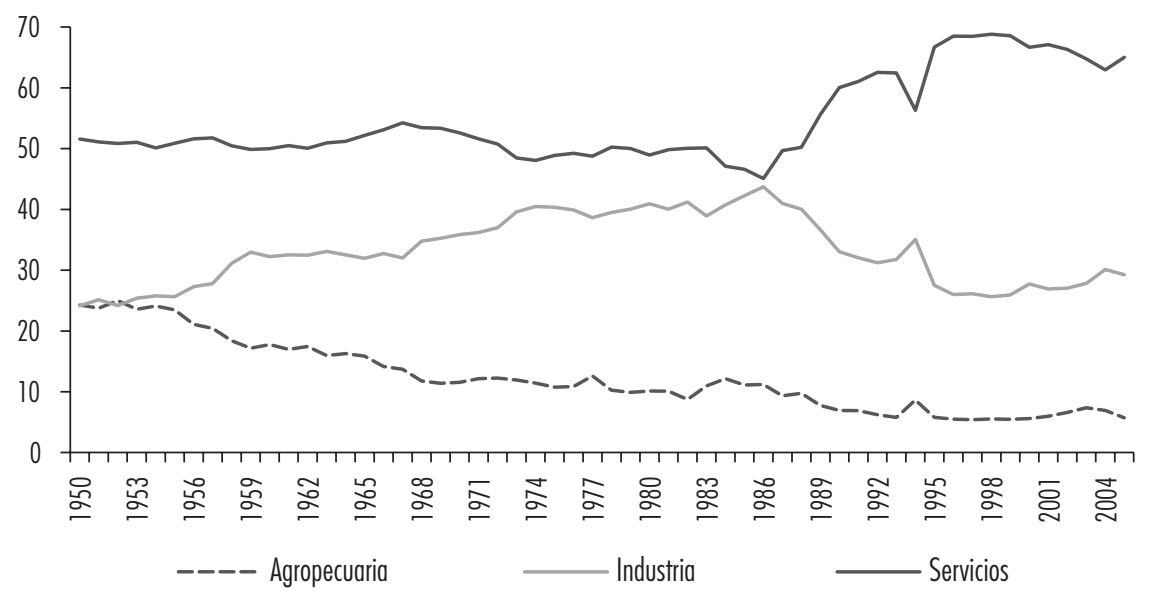

Producción sectorial a precios básicos.

Fuente: Elaboración propia a partir de datos de IBGE (Sistema de Contas Nacionais) disponibles en:

http://seriesestatisticas.ibge.gov.br/lista_tema.aspx?op=0\&no=12

La crisis de la deuda externa de los años ochenta interrumpe drásticamente esta dinámica de crecimiento y transformaciones. Desde entonces la actividad económica crece a un ritmo muy reducido: si en las tres décadas anteriores el PIB se incrementa a una tasa media anual del $7.6 \%$, en los siguientes veinticinco

7 La estructura del empleo registra un cambio análogo: la población activa en el sector primario pasa del $59.9 \%$ al $29.9 \%$, la industrial del $14.2 \%$ al $24.4 \%$ y la de los servicios del $25.9 \%$ al 45.7\% (IBGE, 2003). Utilizamos la población activa porque hasta que en 1967 el IBGE empieza a elaborar la Pesquisa Nacional por Amostra de Domicilios no existen series sistemáticas de empleo. 
años ni siquiera alcanza el 2.2\% anual, de forma que el PIB per cápita apenas crece (lo hace a una tasa del $0.4 \%$ ). El desempeño del sector industrial es aún peor; se incrementa sólo al 1.2\% anual, habiéndolo hecho al $8.7 \%$ al ańo durante las décadas anteriores. ${ }^{8}$ Como consecuencia, desde mediados de los años ochenta y en sólo una década la industria retrocede velozmente, perdiendo alrededor de 20 puntos porcentuales de participación sobre el PIB. Posteriormente, desde 1995 hasta 2005, el peso relativo de las actividades industriales fluctúa entre el 26\% y el 30\%, lo cual, como recoge la Gráfica 1, sitúa al sector en una posición relativa inferior a la de los primeros ańos de los sesenta.

No obstante, ni siquiera durante los treinta ańos de crecimiento "prodigioso" y acelerado ritmo de industrialización, la estructura productiva brasileña es capaz de superar las debilidades que entorpecen su dinámica de acumulación. Y todavía en menor medida cuando la estrategia IsI colapsa. En efecto, a lo largo de todas estas décadas la industria brasileña arrastra sin resolver su deficiencia fundamental: es incapaz de producir bienes de capital en una proporción significativa que permita fortalecer su capacidad interna de acumulación.

El proceso de industrialización registrado en las primeras décadas determina un primer avance en la producción de bienes de equipo (material de transporte e industria mecánica), cuya participación sobre la industria de transformación se eleva desde el $3.9 \%$ al $15.6 \%,{ }^{9}$ si bien hay que considerar que las cifras iniciales eran exiguas, por lo que a la altura de 1980 los bienes de equipo sólo suponen un sexto de la industria de transformación. Además la industria mecánica, núcleo de la producción de maquinaria, no es más que un $7.8 \%$. Y en las décadas siguientes el crecimiento de la producción de bienes de capital es ínfimo, del $0.5 \%$ medio anual entre 1981 y $2005,{ }^{10}$ por lo que la débil especialización brasileña en este tipo de bienes se consolida. Según Carvalho y Kupfer (2007), desde el inicio de los ańos noventa el conjunto de los bienes de equipo mantienen un peso relativo en la industria de transformación sólo 2.5 puntos porcentuales

8 Las tasas de crecimiento medias acumulativas del PIB, el PIB per cápita y el PIB industrial, se calculan a precios de 2011 con datos que estima IPEA a partir de datos de IBGE (Sistema de Contas Nacionais). La serie de datos se puede consultar en: http://www.ipeadata.gov.br/ La producción industrial se aproxima según el valor añadido de la industria de transformación a precios básicos.

9 Estimamos estos datos a partir de datos nominales de producción de la industria de transformación y del PIB obtenidos de IBGE (1990).

10 Cálculos a partir de IBGE (Pesquisa Industrial Mensal, Produção Física), datos disponibles en: http://seriesestatisticas.ibge.gov.br/lista_tema.aspx?op $=0 \&$ no $=8$ 
superior al de 1980, mientras la industria mecánica reduce incluso su participación (oscila entre el $6.5 \%$ y el $7 \%$ ). ${ }^{11}$

Hay que tener en cuenta un dato adicional. Desde 1980 no sólo la industria retrocede posiciones dentro de la producción total, sino que la de transformación también pierde bastante peso relativo: a partir de las mismas fuentes que se indican en el párrafo anterior obtenemos que pasa de alrededor del $78 \%$ entre 1950 y 1980, a perder casi diez puntos porcentuales y situarse en el $69.8 \%$ en 1993, hasta caer en la actualidad casi al 60\%. Este fenómeno supone que las actividades industriales más dinámicas han sido las relacionadas con la minería, la construcción y los servicios básicos (agua, gas, electricidad), y que las ramas manufactureras han tenido un crecimiento bastante lento, aún más acusado en las que fabrican bienes de capital. Por tanto, la especialización en este tipo de productos registra un deterioro superior al que indican los datos anteriores.

Otro rasgo más abunda en la precariedad productiva de la industria brasileña: no sólo produce bienes de capital en proporciones muy reducidas, sino que tampoco los demanda. Así lo revela el hecho de que sus importaciones ocupen un lugar muy minoritario en las compras al exterior, oscilando entre el 10\% y el $20 \%$ sobre las totales. ${ }^{12}$ Por tanto, dado que ni los produce ni los importa, Brasil cuenta con una dotación muy insuficiente de maquinaria y otros equipos, carencia que influye de forma definitiva sobre su capacidad de acumulación.

Como contrapartida, la ampliación de la base industrial se orienta sobre todo a la producción tanto de bienes de consumo duradero (fundamentalmente automóviles y electrodomésticos) como de los bienes intermedios necesarios para su abastecimiento. Estas líneas productivas, pilares del fuerte crecimiento registrado en 1950-1980, concentran hasta la actualidad el grueso de la producción industrial manufacturera. Según Sochaczewski (1993), ya entre 1952 y 1961 la producción de bienes de consumo duradero se incrementa a una tasa anual del 18.2\% (6.6 puntos porcentuales superior a la media industrial). El dinamismo de estas ramas se mantiene hasta finales de los años setenta. Pensemos, por ejemplo, que según datos de la Associaçao Nacional dos

11 Las actividades consideradas por Carvalho y Kupfer (2007) "de mayor contenido tecnológico” nunca superan el 25\% de la producción industrial. También se recomienda Siqueira (2000) y García (2007).

12 Los datos son para 1974-2005 y se obtienen a partir de datos de Funcex. Los datos originales están disponibles en el portal http://www.funcexdata.com.br/indicadores.asp . La proporción de máquinas y equipos importados sobre el total confirman este aspecto: el promedio desde 1970 hasta 2005 es muy reducido, del 23\% (obtenido de la misma fuente). 
Fabricantes de Veículos Automotores (Anfavea) de Brasil entre 1967 y 1980 la producción automovilística nacional se multiplica casi por nueve. ${ }^{13}$ Aunque tras el colapso industrial de los años ochenta el crecimiento en la producción de bienes de consumo duradero se ralentiza en la medida en que no se generan otros cambios, la industria brasileńa mantiene su especialización en este tipo de bienes. Entre 1981 y 2005 registran una tasa de crecimiento anual acumulada del $3.7 \%$, crecimiento que, aunque moderado, casi triplica al de la industria de transformación en su conjunto $(1.4 \%){ }^{14}$

La producción de bienes intermedios es el otro pilar del sector industrial. Su peso relativo en la industria manufacturera se duplica durante las décadas de mayor crecimiento, pasando del $20.2 \%$ al $44.4 \%$ entre 1949 y 1980, manteniéndose después entre el $42 \%$ y el $43.5 \%$ (IBGE, 1990). Lo más relevante es que aunque Brasil muestra cierta especialización en bienes intermedios, la oferta nacional no resulta suficiente para cubrir las necesidades productivas del país. Por ello, la demanda interna es abastecida en buena parte mediante importaciones: las compras en el extranjero de bienes intermedios constituyen un promedio del $51.8 \%$ entre 1974 y 2005 (ver nota 12).

La deficiente especialización productiva del país se confirma, por último, con el hecho de que las ramas industriales más tradicionales (como alimentos y bebidas, textil, vestuario y calzado) nunca dejan de aglutinar una proporción muy relevante de la producción manufacturera. Lejos ya del 75\% que representaba en 1950, en 1980 aún supone cerca del 40\% de la manufactura y en las últimas décadas sigue situándose entre el $33 \%$ y el $35 \%$, una cuota relativa bastante elevada. Pensemos que desde 1981 hasta 2005 la rama de alimentación y bebidas mantiene estable su participación entre el 16\% y el 20\%, mientras que las ramas de textil, confecciones y calzado lo hacen entre el $7 \%$ y el $12 \%$. Un peso específico tan elevado de ramas poco intensivas en capital es consistente con las insuficiencias detectadas en las ramas de bienes de capital y de bienes intermedios que las abastecen.

En síntesis, la composición interna de la industria brasileña revela de forma contundente cuál es la debilidad productiva que arrastra: su incapacidad para producir bienes de capital, a lo que se suma el hecho de que las importaciones de maquinaria y equipos productivos sean también muy reducidas.

13 Exactamente pasa de 7.903 en enero de 1967 a 71.027 en diciembre de 1980. Datos disponibles en http://www.anfavea.com.br/tabelas.html y en el portal de IPEA (http://www.ipeadata. gov.br/ ).

14 Las especificaciones sobre la fuente de estos datos se pueden consular en la nota 10. 
En consecuencia, la especialización de la economía tampoco implica el impulso de una importante producción de bienes intermedios destinados a incorporarse en la producción de bienes de capital. La interacción negativa entre ambos aspectos constituye un límite estructural que afecta de forma fundamental a las posibilidades de desarrollo del país. Este límite, que identificamos al analizar la economía brasileña desde la perspectiva de su especialización productiva, se complementa con otros dos que se aprecian desde la perspectiva de la dotación y la eficiencia de los recursos productivos: la debilidad del stock de capital y la reducida productividad del trabajo.

En primer lugar, cuando se examinan las condiciones del stock de capital se constatan dos hechos simultáneos: su dotación es modesta y su composición técnica muestra una mínima presencia de maquinaria y otros equipos productivos. El stock de capital experimenta un fuerte crecimiento durante el periodo 1950-1980, y después desacelera notablemente su ritmo. Aun así, en el intervalo de 1950-2003 el stock creció a una tasa media cercana al 6\% anual. ${ }^{15}$ Pero dicho stock también incluye la construcción de viviendas residenciales, las infraestructuras públicas, así como la edificación, montajes e instalaciones de todo tipo. Tomando exclusivamente el stock en maquinaria, los hechos delatan una realidad incontestable: entre 1950-2003 el stock apenas ha crecido a una media del $2.5 \%$ anual, y en las tres últimas décadas ni siquiera alcanza el $1 \%$ anual. De esta forma, y a diferencia de lo que caracteriza a los países desarrollados, en Brasil el stock en maquinaria y equipos constituye siempre una parte marginal dentro del stock de capital total. Incluso en los mejores momentos se sitúa sólo en el 31\%, mientras que en los últimos años apenas supone un 18-19\%.

Por consiguiente, la dotación de capital directamente productivo ocupa una posición minoritaria y descendente en un stock total cuya magnitud es modesta. Esta precariedad afecta por partida doble a la productividad: limita el crecimiento del coeficiente capital-trabajo y frena el descenso del coeficiente capital-producto, puesto que queda muy limitada la capacidad de la economía para incorporar y difundir progreso técnico. La conjunción de ambos componentes determina que la productividad del trabajo se incremente con lentitud. Así, y aunque parte de un nivel indiscutiblemente reducido, ni siquiera durante las décadas más dinámicas la productividad del trabajo aumenta a un ritmo potente: diversas estimaciones la sitúan por debajo de una tasa anual del $4 \%$ entre

15 Los datos de crecimiento del stock de capital a los que nos referimos en este apartado los calculamos a partir de las estimaciones del Anexo I de Feu (2004). No es posible ofrecer una serie homogénea para 1950-2005. 
1950 y 1980. Posteriormente, su comportamiento pasa de decepcionante a directamente catastrófico: ronda el $0.2 \%$ anual. ${ }^{16}$

Este aciago resultado deja patente que la reducida eficiencia forma parte del núcleo de elementos que limita a la economía brasileña desde el lado de su capacidad productiva. Aún destaca más cuando se compara su evolución con la registrada por los países desarrollados. Ya en 1950 el PIB por hora trabajada en Brasil constituía 42\% del francés, y 32\% del holandés, mientras que se situaba en niveles mucho más próximos a los de Grecia, Portugal y España, de entre el $85 \%$ y el $95 \%$ (Maddison, 2002). Y el paso de los años profundiza dichas diferencias de manera exagerada. Incluso entre 1950 y 1980, cuando más crece la productividad en Brasil, la distancia con todos los países europeos se ensancha. Y mucho peor es su evolución posterior: en 2005 la productividad brasileña es aproximadamente el 20\% de la que registran Francia y Holanda, y entre un $30 \%$ y un $40 \%$ de la que anotan Grecia, Portugal y Espańa.

\section{INSUFICIENCIA CRÓNICA DE LA DEMANDA}

\section{Debilidad de la inversión}

Las tres principales debilidades productivas señaladas en el apartado se encuentran estrechamente relacionadas con el volumen y la composición de la inversión que se acomete en el país.

Al igual que sucede al analizar la dinámica productiva, la evolución de la inversión nos permite distinguir dos periodos diferenciados. En primer lugar, durante las décadas de 1950-1980 el fuerte crecimiento de la producción está respaldado por un rápido incremento de la formación bruta de capital fijo (FBCF), que registra una tasa de crecimiento anual del $9.4 \%$ y, dentro de ella, de la inversión en maquinaria. No obstante, como ya hemos señalado con otras variables, hay que considerar que Brasil inicia este periodo con unos niveles de inversión ínfimos, de forma que su intenso crecimiento no es óbice para que en términos absolutos la inversión realizada siga siendo muy modesta. Lo comprobamos a continuación.

16 Entre 1950 y 1980 Maddison (2002) estima que la productividad del trabajo crece al 3.7\% anual, y Bonelli (2002) al 3.9\%. Los resultados del primer autor son del $0.18 \%$ anual entre 1981 y 2005, y los del segundo del $0.24 \%$ anual para 1981-2000. Aunque hay discrepancias metodológicas para los noventa, incluso las versiones optimistas son muy moderadas, Rocha (2007) estima un 3.4\% para 1980-2001. 
Por un lado, la tasa de inversión todavía se mantiene en porcentajes reducidos durante los ańos sesenta, $16.1 \%$ en promedio, y alcanza su máximo al término de la década siguiente con un porcentaje medio del $21.4 \%$ del PIB $^{17}$ (Gráfica 2). Por tanto, ni siquiera durante el periodo de mayor intensidad inversora esa cuota alcanza un nivel importante, quedando lejos de los niveles de los países desarrollados durante su periodo de industrialización (25-30\%), sostenidos además durante bastantes años.

Gráfica 2. Tasas de inversión: 1950-2005 (FBCF/PIB \%)

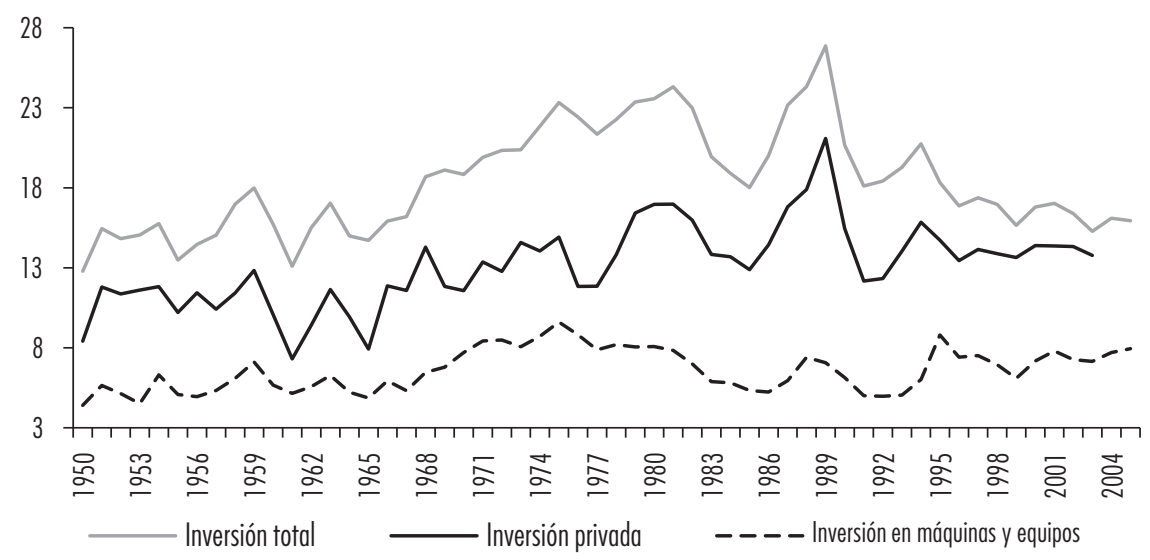

Para calcular la tasa de inversión privada sustraemos la "FBCF de empresas estatales" que el IBGE anota en la partida de "FBCF de empresas y familias". Consiguientemente, incluimos esta partida para calcular la tasa de inversión pública. Fuente: Elaboración propia a partir de datos de IBGE (Sistema de Contas Nacionais), disponibles en: http://seriesestatisticas.ibge.gov.br/lista_tema.aspx?op=0\&no=12 e IPEA.

Por otra parte, la composición de la inversión nos informa de que la parte destinada a equipamiento (maquinaria e instrumentos de trabajo) se mantiene en niveles muy reducidos. La tasa de inversión en máquinas y equipos mantiene un promedio del 5.6\% del PIB durante los ańos cincuenta y sesenta, elevándose hasta el $8.7 \%$ en la década siguiente (datos de IPEA). Es decir, ni siquiera

17 Conviene destacar que entre 1950 y 1980 alrededor de un tercio de la inversión es pública. Las tasas de inversión, así como las participaciones relativas de los restantes componentes de la demanda agregada que se ofrecen en este apartado se calculan a partir de datos de IBGE (Sistema de Contas Nacionais), disponibles en: http://seriesestatisticas.ibge.gov.br/lista_tema. aspx?op=0\&no=12 y del portal de IPEA (http://www.ipeadata.gov.br/). 
en los años en que, según recoge la Gráfica 2, esa inversión se incrementa con más fuerza, supera significativamente el $40 \%$ de la inversión total. ${ }^{18}$ De nuevo el contraste con las experiencias de los países desarrollados es elocuente: tras una primera fase de "capitalización inicial" que exige un alto componente de inversión no residencial en estructuras, es esta inversión la que asume el protagonismo con niveles de participación que sobrepasan el 55-60\% sobre el total.

Ahora bien, si durante aquellas décadas de mayor dinamismo la inversión arrastra las debilidades que acabamos de enumerar, peor aún es su evolución cuando al inicio de los años ochenta se interrumpe la expansión económica: su volumen apenas se incrementa, la tasa de inversión se reduce, y lo mismo sucede con la de equipos productivos.

En efecto, entre 1981 y 2005 el crecimiento de la inversión es insignificante. Comienza reduciéndose a una tasa media anual del $0.7 \%$ hasta 1992 , y posteriormente crece a un ritmo muy reducido del $1.6 \%$ anual. Si atendemos a la evolución de la inversión en máquinas y equipos los resultados no son mucho más alentadores: registra un crecimiento muy leve hasta 1992, del 1.4\% anual, y crece a un 3.4\% anual durante los ańos siguientes. En suma, a lo largo de estos veinticinco ańos la dinámica inversora es desastrosa: la inversión total crece a una media de sólo el $0.5 \%$ anual, mientras que la destinada a equipamientos lo hace al 1.9\%. Este pésimo comportamiento determina que desde los años noventa hasta la actualidad, aun tratándose de un periodo en el cual el PIB también crece a un ritmo lento, la tasa de inversión nunca alcance una cuota destacada, con un promedio en torno al 19\%, mientras que la tasa de inversión en equipos se mantiene en torno al $6.5 \% .^{19}$

En síntesis, durante las décadas de mayor crecimiento y transformaciones económicas, entre 1950 y 1980, Brasil no supera las restricciones estructurales que limitan su dinámica inversora, mientras que en el siguiente cuarto de siglo dichas restricciones se agudizan. Por ello resulta evidente que la economía brasileña arrastra una deficiencia crónica de demanda de inversión, que aún es más acuciante en el caso de la inversión en maquinaria y otros equipos productivos

18 Es paradigmático que en este periodo la industria mecánica sólo concentre el 7.2\% del total de inversiones destinadas a la industria de transformación. Cálculos para 1959-1980 a partir de IBGE (1990).

19 Se obtienen las tasas de crecimiento real calculando las tasas promedio de crecimiento acumulativo de las series a las que nos referimos en la nota 17 a precios constantes de 2005 (facilitados por IPEA). 


\section{Estrechez y segmentación de la demanda de consumo}

La estrechez del mercado interno que se constata para la inversión, se refuerza con la que presenta el consumo privado. Entre 1950 y 1980, el consumo de las familias brasileńas se incrementa a una tasa media anual del $7 \%{ }^{20}$ convirtiéndose, junto con la inversión y el consumo realizados por el Estado, en los motores del potente crecimiento registrado. Por el contrario, a partir de entonces el consumo privado evoluciona lentamente, de forma que entre 1981 y 2005 anota un crecimiento medio muy leve, del $1.4 \%$ anual. Por consiguiente, considerando que los niveles iniciales de consumo son ínfimos y que su ritmo de crecimiento queda bruscamente interrumpido a comienzos de los ochenta, cabe afirmar que el primer rasgo que pone de manifiesto la demanda de bienes y servicios de consumo es la reducida magnitud de su mercado interno.

Adicionalmente, como segundo rasgo, si el consumo final evoluciona de forma más dinámica es porque el consumo realizado por el Estado se incrementa a un ritmo importante y sostenido. El consumo público no sólo aumenta a una tasa próxima a la del pis durante las décadas de mayor dinamismo (del 6.6\%), sino que también registra un incremento importante (del 5.3\% anual) entre 1981 y 2005. Es así que a lo largo de los cincuenta y cinco años considerados, el consumo del Estado crece a un ritmo superior al 6\% anual, es decir, bastante más de lo que lo hace el consumo privado.

El otro rasgo importante que revela el comportamiento del consumo privado es que se caracteriza por un grado de segmentación muy elevado. La composición del consumo de las familias delata una estructura muy polarizada, que se corresponde con dos patrones de consumo antagónicos: uno basado casi exclusivamente en bienes de subsistencia y otro de muy elevada elasticidad-renta.

Es sintomático que una parte relevante del consumo privado se componga de bienes duraderos, así como que una parte significativa sean bienes y servicios que sólo pueden ser adquiridos con niveles de renta medio-altos y altos. Así lo confirma el hecho de que los mercados de automóviles y de electrodomésticos sean de los más dinámicos. En realidad, es el crecimiento de dichos mercados lo que explica la expansión del consumo privado durante las décadas de la industrialización, y aunque se desacelera a partir de los años ochenta, siguen

20 Se recuerda que las tasas de crecimiento de los componentes de la demanda agregada -consumo privado y consumo público, en este apartado- se calculan a partir de las series de IBGE (Sistema de Contas Nacionais), disponibles en: http://seriesestatisticas.ibge.gov.br/lista_ tema.aspx?op=0\&no=12 y del portal de IPEA (http://www.ipeadata.gov.br). 
destacando posteriormente. En efecto, durante los años setenta las ventas de automóviles se disparan, incrementándose a una tasa anual del $13.1 \%$, de forma que a finales de la década se vendían en Brasil aproximadamente 800,000 coches al año (Anfavea, nota 13). En esos años el consumo de electrodomésticos también crece espectacularmente: el número de ventiladores vendidos lo hace al $15.1 \%$ anual, el de aparatos de radio al 14.2\%, los equipos de aire acondicionado al $14 \%$, las televisiones vendidas se incrementan al $13.6 \%$ cada año, y los frigoríficos al $12 \%$. Pensemos que en los últimos ańos de los setenta los brasileños ya compran alrededor de 2.8 millones de televisores, 5 millones de equipos de radio y 2 millones de frigoríficos al año. ${ }^{21} \mathrm{El}$ consumo de este tipo de bienes se reduce considerablemente desde los ochenta, pero sigue manteniendo un ritmo de crecimiento muy superior al del consumo privado total.

Al mismo tiempo, ciertos bienes de lujo también ocupan un lugar relevante en el mercado de consumo. Nos referimos a joyas, moda de alta costura, automóviles y equipos electrónicos de gama superior, yates y helicópteros de uso personal, etcétera. A título de ejemplos, cabe destacar que Brasil es el segundo país del mundo en ventas de plumas Montblanc y el noveno en ventas de coches de la marca Ferrari (Cauduro, 2004: 18). La empresa francesa Louis Vuitton, especializada en alta costura y complementos de lujo, tiene en São Paulo su quinta tienda a escala mundial por volumen de facturación (MCF Consultoria \& Conhecimento y Gfk Indicator, 2009). Aunque la demanda de este tipo de bienes se sostiene, según analizamos en el apartado siguiente, por grupos sociales que representan una proporción muy reducida de la población, la dimensión del país determina que el mercado de lujo alcance un tamaño muy importante, que en la actualidad se calcula que mueve 5,000 millones de dólares anuales. No en vano Brasil concentra el $70 \%$ del consumo de lujo de América Latina, y se sitúa en el segundo puesto dentro del ranking mundial de mercados de lujo por potencial de crecimiento (Ibídem). ${ }^{22}$

En definitiva, la estrechez y segmentación del consumo privado también se relacionan con una especialización productiva que se decanta por las ramas de

21 Los datos son de Abinee (Asociaçao Brasileira de Indústria Elétrica e Eletrônica), pero se encuentran con más facilidad en el portal de IPEA (http://www.ipeadata.gov.br/).

22 Es muy ilustrativo el análisis realizado por un ejecutivo de Bang \& Olufsen, empresa fabricante de equipos de audio y vídeo de alto nivel: aunque calculan que la población brasileña que accede a sus productos es inferior al $1 \%$ sobre la total, ese mercado potencial equivale aproximadamente a la mitad del de Dinamarca, país de origen del grupo empresarial. Según concluye, "Brasil es un país tan grande que es casi imposible no ganar dinero" (revista Veja, 24 de julio de 2002, p. 28). 
bienes de consumo dentro de su estructura industrial, y que tiene su pilar fundamental en la producción de bienes de consumo duradero.

\section{DISTRIBUCIÓN DE LA RENTA MUY POLARIZADA}

Según se plantea en el primer apartado, la dimensión y la morfología de la demanda interna mantiene una estrecha relación con las características y la evolución de la distribución de la renta nacional.

Abordando en primer lugar la relación entre la distribución de la renta en Brasil y la demanda de consumo en su volumen y composición, hay que comenzar destacando la baja participación de las rentas salariales sobre la renta global del país. En promedio, la masa salarial respecto del PIв mantiene un nivel muy reducido, inferior al $43 \%$, mientras que en los países desarrollados los salarios registran una participación muy superior, situada según los casos entre el 55\% y el 60\%. ${ }^{23}$ Como vemos en la Gráfica 3, a mediados de los años cincuenta la masa salarial alcanza un máximo puntual de participación del $48 \%$, y tras mantenerse los siguientes años entre dicho valor y el $45 \%$, inicia un hundimiento estrepitoso, de forma que en la década de los setenta la participación salarial arroja un promedio de sólo el 40.1\%. Aunque se recupera durante los ańos ochenta y la primera mitad de los noventa (cuando la renta global permanece prácticamente estancada), después sobreviene un derrumbe de magnitud muy superior: en poco más de una década las rentas del trabajo pierden 12.3 puntos porcentuales sobre la renta total, de forma que en 2005 registran la participación mínima de toda la serie histórica, situándose alrededor del 36\%.

El notable descenso que muestra la línea de tendencia de la Gráfica 3 no deja lugar a dudas acerca de los dos hechos fundamentales: la pérdida de participación de la retribución salarial en la renta global y su bajísimo nivel relativo desde mediados de los años noventa. Además, dicha evolución permite distinguir dos periodos diferenciados. Durante las tres primeras décadas, y a pesar de la intensa desaceleración que se produce entre finales de los años sesenta y los primeros de los setenta, la masa salarial se incrementa a una tasa media anual del 7.1\%,

23 Los datos de masa salarial sobre el pIB que ofrecemos en este apartado se obtienen siguiendo la metodología propuesta por Marquetti (2003) y Bruno (2007) a partir de datos del mismo trabajo de Marquetti y de IBGE (Sistema de Contas Nacionais) a los que se ha accedido a través del portal de IPEA (http://www.ipeadata.gov.br/ ). 
similar a la que registran el PIB y el consumo privado. Así, aunque dicho crecimiento se ve parcialmente determinado por el rápido aumento del número de asalariados (la población activa urbana crece al 5\% anual), cabe estimar que en estas décadas existe también cierta mejora en el salario unitario. ${ }^{24}$

Gráfica 3. Masa salarial: 1950-2005

(\% del PIB)

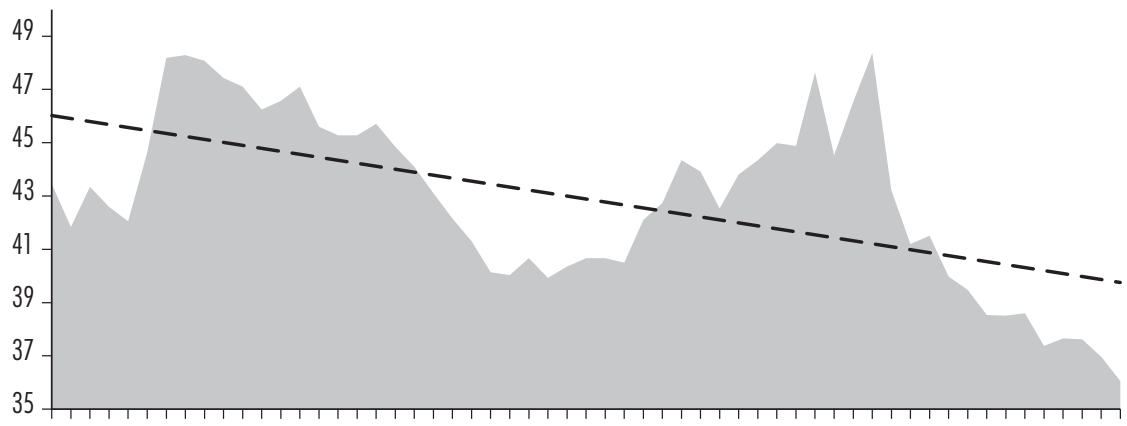

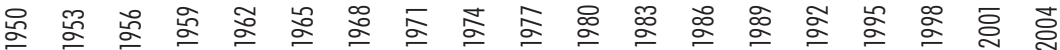

Fuente: Elaboración propia siguiendo la metodología propuesta por Marquetti (2003) y Bruno (2007) a partir de datos del trabajo de Marquetti y de IBGE (Sistema de Contas Nacionais). La línea de tendencia es lineal.

La situación se revierte a partir de los años ochenta. De una parte, el crecimiento de la masa salarial se desacelera bruscamente: entre 1981 y 2005 apenas crece al $1.8 \%$ anual. ${ }^{25}$ De ese modo, aunque la creación de empleo también reduce su ritmo de crecimiento, teniendo en cuenta que según los datos del censo oficial el conjunto de la población activa urbana se incrementa

24 Hasta el golpe de Estado de 1964, el denominado pacto populista representado por Vargas supone una mejora económica ostensible para el nuevo grupo de trabajadores urbanos industriales: en los años cincuenta el salario mínimo real se multiplica por tres. Consultar Lessa (1998) y Marques y Rego (2003).

25 Los picos que recoge la Gráfica en 1989 y 1995 no se corresponden con un crecimiento nominal de la masa salarial, sino con grandes caídas en los índices de inflación como consecuencia de sendos planes de estabilización que tienen efectos bruscos en el corto plazo (Plan Verao y Plan Real). 
al 3.4\% anual, cabe deducir que el empleo asalariado sigue aumentando a un ritmo significativo y, por tanto, que el salario unitario prácticamente no crece, o incluso desciende. La evolución del salario mínimo legal, el único que permite establecer una serie salarial homogénea desde 1950, confirma esa estimación. ${ }^{26}$ Tras incrementarse con fuerza en términos reales durante los años cincuenta y sesenta, retrocede a finales de esa década y comienzos de la siguiente. La posterior recuperación queda truncada al inicio de la década de los ochenta y vuelve a acusar una caída fortísima, de modo que al comenzar los años noventa el salario mínimo brasileńo anota su valor mínimo, siendo aproximadamente un 38\% del vigente durante la era Vargas. Aunque posteriormente mejora levemente, todavía en 2005 su valor equivale al de principios de los años ochenta. ${ }^{27}$ Además, hay que considerar que a partir de la década de los ochenta el mercado de trabajo brasileño se deteriora velozmente. La reducción de los ámbitos laborales más estructurados (la industria, incluida la producción estatal), la aparición del desempleo urbano como fenómeno masivo y el incremento de la informalidad, entre otros factores, determinan que la dispersión de los ingresos salariales se incremente, lo que nos indica que existen franjas importantes de trabajadores que, con toda seguridad, no experimentan ningún crecimiento de sus salarios reales (Medialdea, 2009).

En definitiva, teniendo en cuenta que una gran parte de la población brasileña es asalariada y que la considerable parte de personas que trabajan en la economía informal recibe ingresos también muy reducidos, el patrón de distribución de renta vigente determina que la inmensa mayoría de las familias registre un crecimiento muy lento de sus ingresos y, consiguientemente, mantenga una capacidad de consumo muy restringida, que en grupos sociales muy amplios se sitúa en niveles próximos a los de subsistencia. En esa medida, el reparto de la renta entre capital y trabajo se convierte en un límite estructural que da lugar a la estrechez del mercado de consumo.

Simultáneamente, ese desigual reparto es también el que explica la dualidad del mercado de consumo, ya que mientras el ingreso disponible de la mayoría de los hogares crece con lentitud y restringe su capacidad de compra a una

26 La evolución del salario mínimo es indicativa porque las cláusulas de ajuste salarial, parte de los salarios informales y algunas prestaciones sociales se fijan tomándolo como referencia.

27 El valor real del salario mínimo se toma de IPEA (http://www.ipeadata.gov.br/), que deflacta los valores nominales con el ICV (Índice do Custo de Vida) hasta 1979 y con el INPC (Índice Nacional de Preços ao Consumidor) a partir de ese año. 
cesta de bienes y servicios básicos, una parte reducida de los hogares concentra proporciones muy elevadas de ingreso disponible y tiene una alta propensión al consumo de bienes de notable elasticidad-renta. Para constatarlo, es preciso complementar los datos de distribución funcional de la renta con la distribución del ingreso en términos personales. En efecto, Brasil es uno de los países del mundo cuya desigualdad de la renta es más exagerada. ${ }^{28}$ Por ejemplo su índice Gini, que se sitúa en torno a 0.6, es de los más altos de Latinoamérica, siendo ésta la región con mayor desigualdad a escala mundial. ${ }^{29}$

Otros datos abundan en la misma dirección: mientras que el 10\% más rico de población acapara el $47.8 \%$ del ingreso disponible, un grupo cinco veces más numeroso, el del $50 \%$ con menos renta, vive con un ingreso casi cuatro veces inferior, el $12.5 \%$ sobre el total. ${ }^{30}$ Esto significa también que el $1 \%$ de la población compuesto por el estrato de ingresos más alto detenta una proporción de la renta $(14 \%)$ que supera a la que tiene ese $50 \%$ más pobre. También hay que destacar que el $20 \%$ de población de menores ingresos, es decir, una cantidad muy grande de personas (en la actualidad ronda los 40 millones), apenas capta el $2.4 \%$ de la renta disponible, lo que les condena a vivir en condiciones de extrema precariedad, con niveles muy reducidos de consumo. Según ilustra la Gráfica 4, más allá de oscilaciones irrelevantes, esta brutal radiografía socioeconómica se mantiene estable desde el inicio de las series disponibles hasta 2005.

En el otro extremo está esa élite del 10\% más rico, junto con otras franjas formadas por profesionales calificados, propietarios de negocios de cierto tamaño, asalariados de mayor categoría y otros grupos sociales, cuya capacidad de consumo es de apreciable a extraordinaria, según su nivel de ingresos. Ellos son los que sostienen la demanda de bienes duraderos de gamas superiores, así como otros bienes y servicios, que en parte se abastecen con producción interna y en parte deben ser importados. Así, la consolidación de un mercado dinámico de bienes de consumo duradero se sostiene no sólo por las familias más ricas sino también por esos segmentos sociales intermedios, siendo aquella élite rica la que genera la demanda del dinámico mercado de bienes

28 Sobre el origen histórico de la extrema desigualdad brasileña se puede consultar Furtado (1959), Dos Santos (1994) y Lessa (2005).

29 Según el PNUd este índice toma un valor de 59.0 en 1985, 60.6 en 1990 y 59.2 en 1995 (ver: http://hdrstats.undp.org/es/indicadores/67106.html )

30 Los datos de este párrafo se tomaron de IPEA (Diretoria de Estudos Sociais), que los calcula a partir de la Pesquisa Nacional por Amostra de Domicílios elaborada por el IBGE. Se pueden consultar en el portal: http://www.ipeadata.gov.br/ 
de lujo. Es importante considerar que aunque se trata de un grupo social y de un patrón de consumo minoritarios, la magnitud continental de Brasil hace que en cifras absolutas el número de consumidores y el valor de sus compras sean muy elevadas. De ese modo, suponiendo que ese grupo represente sólo a un $20 \%$ de la población, eso significa que en 1950 estaba compuesto por unos 10 millones de personas y en 2005 por 38 millones, las cuales concentran el $65 \%$ de la renta disponible, ${ }^{31}$ lo que implica que su mercado es bastante amplio. De la misma forma, aunque según las estimaciones, sólo entre un $1 \%$ y un $2.5 \%$ de la población accede al mercado de bienes de lujo, actualmente esto significa una demanda de entre 2 y 5 millones de personas.

Gráfica 4. Distribución personal de la renta: 1976-2005

(\% de la renta total)

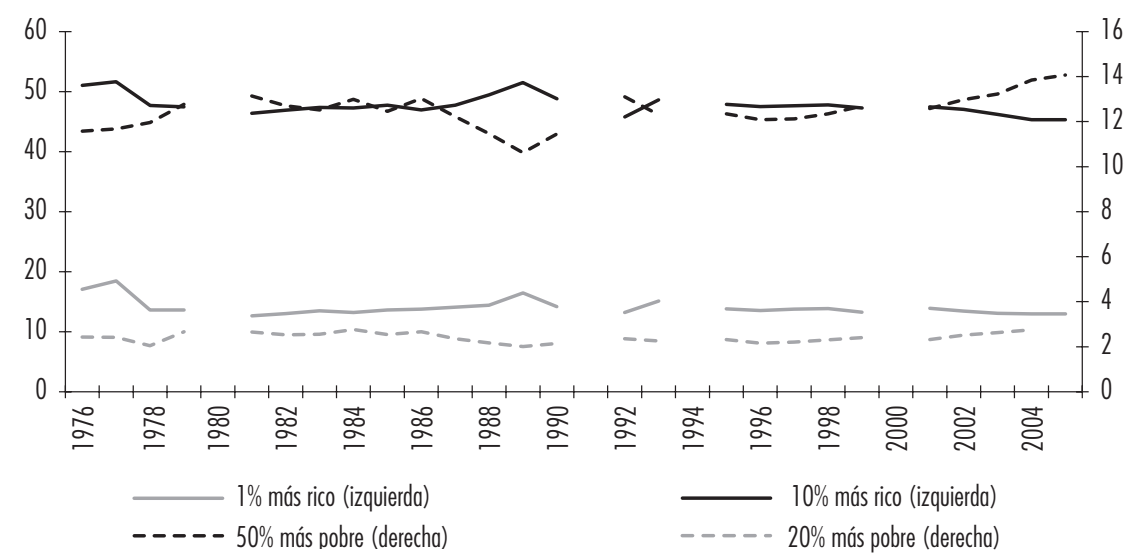

Fuente: Datos de IPEA (Diretoria de Estudos Sociais) calculados a partir de la Pesquisa Nacional por Amostra de Domicílios elaborada por el BBGE. Se pueden consultar en el portal: hittp://www.ipeadata.gov.br/

La relación entre la distribución de la renta y la demanda interna no se ciñe exclusivamente a los vínculos respecto a la demanda de consumo. También afecta al comportamiento de otro componente decisivo de la demanda: la inversión empresarial. El reparto del beneficio entre las diferentes facciones capitalistas explica la debilidad de la inversión productiva. Si la inversión depende fundamentalmente de las expectativas de rentabilidad, el simple repaso de los rasgos que caracterizan a las facciones

31 Promedio para 1976-2005 calculado a partir de IPEA (Diretoria de Estudos Sociais). Se puede consultar en el portal: http://www.ipeadata.gov.br/ 
del capital que concentran el poder económico y político del país, evidencia condiciones adversas para el crecimiento sostenido de la inversión.

En primer lugar los terratenientes, que hegemonizaron el modelo primario exportador, quedan relegados como facción capitalista según avanza la industrialización impulsada por la estrategia ISI. Algunas familias de hacendados conservan cierta presencia en el sector agroalimentario (soya, carne roja, café, caña de azúcar, algodón) y de recursos naturales. En cualquier caso, dichas actividades industriales, orientadas a la exportación, mantienen una conexión muy débil con el conjunto del tejido industrial.

Las empresas nacionales, en su condición de lo que se denominó "la pata débil del tripiê", juegan siempre un papel subsidiario en la dinámica industrial del país, concentrándose en actividades productivas y comerciales de tipo tradicional y en actividades de abastecimiento a las empresas extranjeras. Desde finales de los años setenta, con un grado de penetración extranjera en el sector industrial muy elevado, el control del capital nacional se concentra en las ramas de vestuario y calzado, alimentos y bebidas, actividades de edición y gráficas, o mobiliario (Gonçalves, 1999: 116-118). Ese tipo de bienes de consumo masivo es el único segmento productivo rentable al que accede el capital brasileño: el mercado de bienes de consumo más sofisticado está controlado por las empresas extranjeras, mientras que la precariedad de la estructura productiva determina la inexistencia de rentabilidad suficiente en la producción de bienes de capital. Asimismo, la producción de bienes intermedios y de servicios básicos se lleva a cabo fundamentalmente a través de las empresas estatales, y pasa posteriormente a manos del capital extranjero mediante las privatizaciones. En consecuencia, su propia especialización en un segmento de escaso nivel de capitalización y dinamismo tecnológico, y cuyo potencial de crecimiento viene limitado por la exigua capacidad de consumo de la mayoría de la población, determina niveles de inversión limitados, generando una demanda muy escasa de bienes de producción.

En tercer lugar, el capital financiero nacional no toma protagonismo hasta los años ochenta. Hasta entonces es el Estado, a través de las empresas productivas estatales y de la banca estatal, el que asume el protagonismo del proceso de industrialización. Es a partir de la crisis de la deuda externa y en el contexto de las políticas de ajuste cuando se conforma una banca privada de cierto relieve. Ésta ya nace con una elevada propensión rentista que se alimenta del negocio financiero que posibilitan los títulos de deuda indexada emitidos por el Estado. En la década de los noventa, en un contexto de liberalización financiera, política monetaria favorable a los acreedores, privatizaciones y entrada masiva de capital extranjero, se produce la consoli- 
dación definitiva de esta facción capitalista, cuya contribución a la financiación empresarial y el consumo privado es muy reducida (Medialdea, 2010).

Por último, en el transcurso del tiempo el capital internacional participa crecientemente en la dinámica económica del país. Desde los años setenta controla la producción de buena parte de los bienes de consumo duradero, es decir, los productos que cuentan con una demanda más amplia, lo cual le permite obtener beneficios importantes y estimula la dinámica inversora. De esta forma, aunque el capital extranjero expatria una parte importante de las rentas obtenidas, también realiza reinversiones considerables y favorece con ello, hasta finales de los años setenta, el crecimiento de la inversión y la producción. Sin embargo, tras el colapso de la estrategia ISI, la reinversión en el país de los beneficios obtenidos por el capital extranjero disminuye notablemente. Los estímulos a la inversión mejoran otra vez durante los ańos noventa, gracias al nuevo contexto de liberalización, apertura externa y grandes privatizaciones en sectores muy atractivos.

En síntesis, la posición que ocupan los distintos tipos de empresarios en la actividad económica se convierte en un factor desfavorable para el crecimiento sostenido de la inversión y, por ello, para la transformación de la especialización productiva, el mayor progreso tecnológico, el aumento de la productividad y otros rasgos que requiere la dinámica de acumulación de una economía desarrollada. Es por ello que, cuando el Estado (que sostiene el crecimiento registrado entre 1950 y 1980) se retira de la actividad económica, los distintos grupos de capital nacional y extranjero que detentan, con distinto grado, el poder económico, mantienen una dinámica inversora débil.

\section{CONCLUSIÓN}

La conjunción de los elementos analizados en este artículo se plasma en la morfología y el comportamiento del ahorro interno. Por una parte, la debilidad inversora restringe la capacidad de acumulación de Brasil y con ello sus posibilidades de crecimiento. De forma simultánea, el esquema de distribución de la renta refuerza la debilidad de la inversión, a la vez que determina la estrechez y la dualidad del mercado interno de consumo. Como resultado, el ahorro generado internamente es sistemáticamente bajo, de modo que es insuficiente por sí mismo para permitir un incremento potente y sostenido de la inversión. En definitiva, la insuficiencia de ahorro se convierte en un obstáculo estructural adicional para la economía porque restringe severamente las posibilidades de que la inversión se financie internamente. 
Dicha restricción opera a través de dos mecanismos. De una parte, como el nivel de renta nacional es reducido y además se distribuye de forma que el grueso de ésta es captado por grupos sociales que no tienen incentivos para invertir, la mayor parte de la renta se dedica al consumo, lo cual reduce el margen para la formación de ahorro. Por otro lado, si el contexto no favorece que ese reducido ahorro disponible se canalice hacia grupos sociales con propensión a invertir, el ahorro saldrá del país o permanecerá en él sin comprometerse con la actividad productiva.

La doble restricción del ahorro, en cuanto a su magnitud reducida y a su escasa movilización hacia destinos productivos, es una constante de la economía brasileña que adopta modalidades distintas a lo largo del tiempo. El periodo de crecimiento de 1950-1980 aporta evidencias sobre cómo las vías alternativas con las que se trata de "atajar" esta restricción, en ausencia de transformaciones económicas y sociales que desactiven los límites estructurales al desarrollo analizados, acaban por ser insostenibles. De un lado, la opción por la financiación inflacionista del crecimiento hasta mediados de 1960 (Giambagi et al., 2005), y de otro, el recurso a créditos bancarios internacionales a lo largo de los años setenta (Carneiro, 2002), son prueba de ello. Cabría plantearse, a modo de reflexión, que pretender incidir sobre los límites estructurales al desarrollo, sin acometer las profundas transformaciones que requeriría liberarse de ellos deriva, invariablemente, en la aparición de problemáticas adicionales (como lo fueron un nivel de inflación insostenible a mediados de los años sesenta o el estallido de la crisis de deuda externa a principios de los ochenta).

En definitiva, la experiencia brasileña correspondiente al periodo 19502005, analizada sintéticamente en este artículo a través de sus principales relaciones macroeconómicas, pone de manifiesto que a lo largo de estas décadas la economía de Brasil permanece sometida a restricciones de naturaleza estructural que interactúan entre sí y consolidan su subdesarrollo.

\section{BIBLIOGRAFÍA}

Bonelli, Regis, Labor Productivity in Brazil During the 1990 's, IPEA, Texto para Discussão, num. 906, 2002.

Bruno, Miguel, “Transição Demográfica e Regime de Acumulação Financeirizado no Brasil: "Bônus" ou "Ônus" para a Previdência Social?", Centro de Estudos Sindicais e de Economia do Trabalho, Carta Social e do Trabalho, num. 7, 2007. 
Carneiro, Ricardo, Desenvolvimento em Crise. A Economia Brasileira no ultimo Quarto do Século XX, São Paulo, Editora Unesp, 2002.

Carvalho, Laura B. y David A. Kupfer, Transiçāo Estrutural da Indústria Brasileira da Diversificação para a Especialização, Anais do xxxv Encontro Nacional de Economia da ANPEC, 2007.

Cauduro, A., Valores e Significados do Consumo de Produtos de Luxo, Tesina de Mestrado, Universidade Federal do Rio Grande do Sul, 2004.

CEPAL (Ed.), Cincuenta años de pensamiento en la CEPAL: textos seleccionados, Santiago de Chile, FCE, 1998.

Dos Santos, Theotonio, Evolução Histórica do Brasil. Da Colonia á Crise da "Nova República", Petrópolis, Vozes, 1994.

Feu, Aumara, "Evaluation of Capital Productivity in Brazil in the xx Century", en Economy \& Energy, num. 23, abril-mayo de 2004.

Furtado, Celso, Formação Económica do Brasil, Río de Janeiro, FCE, 1959.

García, Carmen, Indústria e Capital Estrangeiro no Brasil do pós-guerra aos anos 2000, Tesis Doctoral, Universidade Federal do Rio de Janeiro (UfRJ), 2007.

Giambiagi, Fabio, André Villela y Lavínia Barros de Castro (org.), Economia Brasileira Contemporánea (1945-2004), Río de Janeiro Campus, 2005.

Gonçalves, Reinaldo, Globalização e Desnacionalização, S. Paulo, Paz e Terra, 1999.

Griffin, Keith, Alternative Strategies for Economic Development, Londres, MacMillan Press, 1989.

IBGE, Estatísticas Históricas do Brasil, Río de Janeiro, IBGE, 1990.

IBGE, Estatísticas do Século XX, Río de Janeiro, IBGE, 2003.

Kalecki, Michal, Ensayos escogidos sobre dinámica de la economía capitalista, 1933-1970, México, FCE, 1977.

Lessa, Carlos (org.), Enciclopédia da Brasilidade: auto-estima em verde e amarelo, Río de Janeiro, BNDEs, 2005.

Maddison, Angus, La economía mundial. Una perspectiva milenaria, Madrid, Ediciones Mundi-Prensa, ocDE, 2002.

Marques, Rosa Maria y José Márcio Rego (Eds.), Economia Brasileira, São Paulo, Saraiva, 2003.

Marquetti, Adalmir, Nota Metodológica sobre as Informaçóes Estatísticas Utilizadas na Análise do Padrão de Progresso Técnico na Economia Brasileira, 19501980, Instituto de Economia/puC-Rs, Rio Grande do Sul, 2003.

MCF Consultoria \& Conhecimento y Gfk Indicator, O Mercado de Luxo no Brasil, ano II, Mimeo, 2009. 
Medialdea, Bibiana, “Ajuste salarial en Brasil: 1994-2007”, en Álvarez Peralta et al, Ajuste y Salario. Las consecuencias del neoliberalismo en América Latina y Estados Unidos, pp. 81-110, Fondo de Cultura Económica, Madrid, 2009.

- Subdesarrollo, capital extranjero y financiarización: la trampa financiera de la economía brasileña, Madrid, Tesis Doctoral en la UCM, 2010.

Palazuelos, Enrique y Rafael Fernández, "Demand, Employment and Labour Productivity in the European Economies", en Structural Change and Economics Dynamics, v. 20, num. 1, marzo de 2009, pp. 1-15.

Puerto, Luis Miguel, Economia para el desarrollo. Lecturas desde una perspectiva crítica, Madrid, Libros de la Catarata, 2008.

Rocha, Frederico, "Produtividade do Trabalho e Mudança Estrutural nas Indústrias Brasileiras Extrativa e de Transformação, 1970-2001", en Revista de Economía Política, v. 27, num. 2, abril-junio de 2007, pp. 221-241.

Rodríguez, Octavio (Ed.), La teoría del subdesarrollo de la CEPAL, México, Siglo XXI, 1993.

Siqueira, Tagore, "A Indústria Brasileira nos Últimos 16 anos do Século 20: 1985-2000”, en Revista do BNDES, v. 7, num. 14, 2000, pp. 55-105.

Sochaczewski, Antonio Cláudio, Desenvolvimento Económico e Financiero do Brasil: 1952-1968, São Paulo, Trajetória Cultural, 1993.

Szirmay, Adam, The Dynamics of Socio-Economic Development, Cambridge, Cambridge University Press, 2005.

Tavares, Maria da Conceição, "Auge e Declínio do Processo de Substituição de Importaçôes no Brasil", Boletín Económico de América Latina, 1964 pp. $1-62$.

Todaro, Michael, El desarrollo económico del Tercer Mundo, Madrid, Alianza, 1985. 